Supporting Information

\title{
FleXert: a soft, actuatable multi-well plate insert for cell culture under stretch
}

Sara Correia Carreira ${ }^{1 *}$, Majid Taghavi, Elizabeth Pavez Lorie ${ }^{3}$, Jonathan Rossiter2*

${ }^{1}$ University of Bristol, School of Cellular and Molecular Medicine, University Walk, Bristol BS8 1TD, United Kingdom

2University of Bristol, Bristol Robotics Laboratory, T Block, Frenchay Campus, Coldharbour Lane, Bristol BS16 1QY, United Kingdom

${ }^{3}$ Leibniz Research Institute for Environmental Medicine, Auf'm Hennekamp 50, 40225 Düsseldorf, Germany

*corresponding authors; email: s.carreira@bristol.ac.uk,

jonathan.rossiter@bristol.ac.uk

Content: 2 pages, 3 figures. 


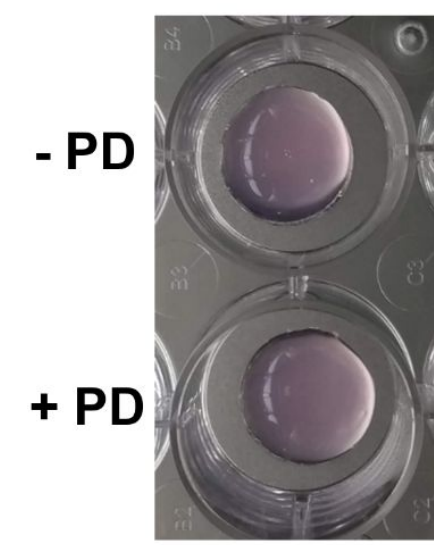

d0

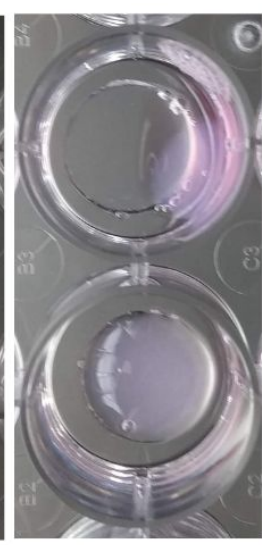

d1

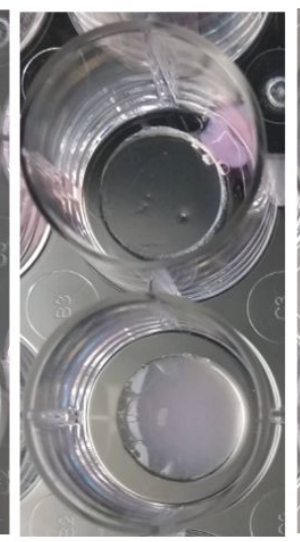

d3

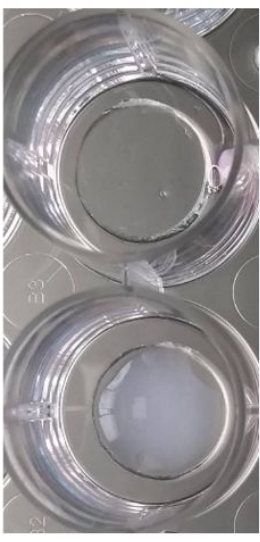

d6

Figure S1: Anchoring a $0.2 \%$ collagen gel to PDMS using polydopamine (PD) coating. Human dermal fibroblasts were suspended in collagen gels and placed on uncoated or polydopamine coated PDMS discs inside a 24 well plate. After $24 \mathrm{~h}$ culture (d1), gels had delaminated from the uncoated PDMS surface, whereas gels remained anchored to the PD coated PDMS for at least 6 days in culture (d6).
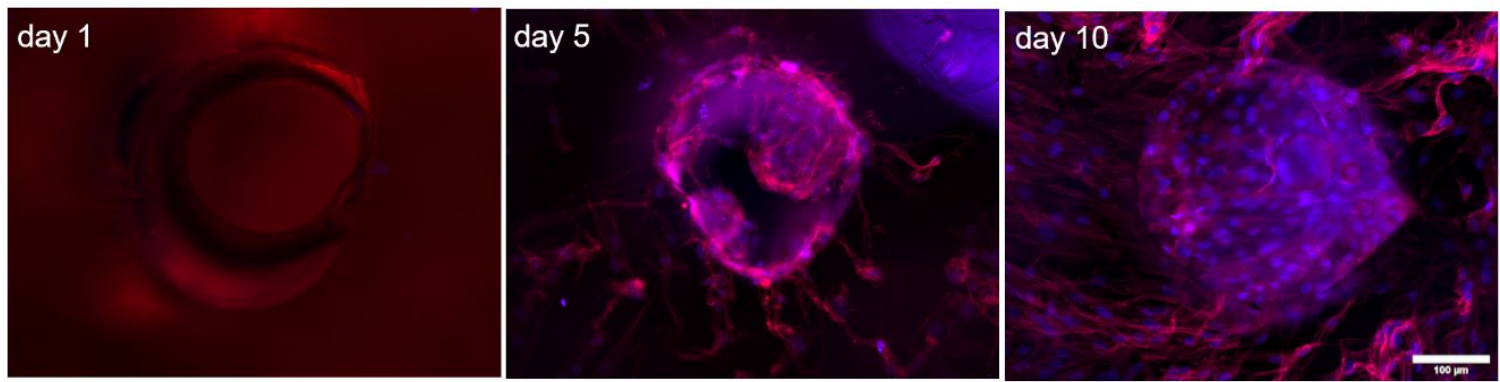

Figure S2: Imaging the underside of FleXerts coated with polydopamine and collagen and seeded with $10^{5}$ human dermal fibroblasts after 1, 5 and 10 days in culture. F-actin stained with phalloidin (red) and nuclei with DAPI (blue). On day 1, no cells are visible on the underside of the porous membrane (cells on the top visible as an out-of-focus red haze). On day 5, cells have grown through the pore and colonised the underside of the membrane. On day 10, cells have filled the pore and extensively covered the underside of the FleXert membrane. Scale bar: $100 \mu \mathrm{m}$. 

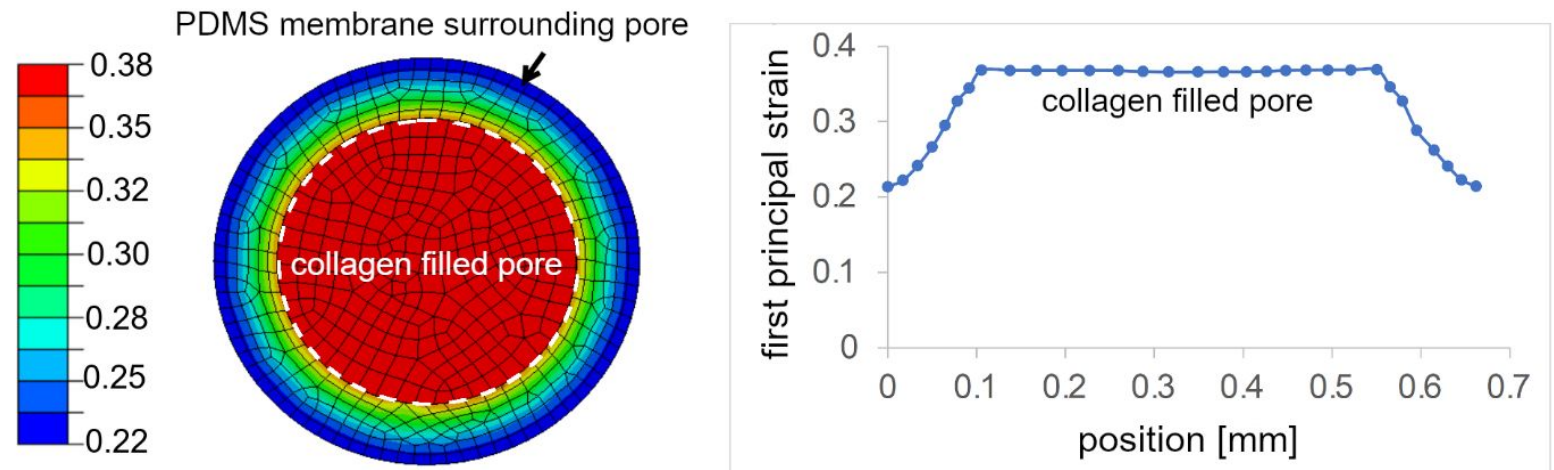

Figure S3: Finite element analysis of a collagen filled pore under actuation (-0.65 bar applied). Cells growing on the PDMS membrane surrounding the pore experience a strain gradient, while cells growing on the collagen layer spanning the pore experience uniform strain. The magnitude of strain is slightly higher for cells growing on the collagen compared to cells growing on PDMS. 